\title{
Schrödinger-type identity to the existence and uniqueness of a solution to the stationary Schrödinger equation
}

Delin Sun ${ }^{1 *}$

"Correspondence:

dlsun12@foxmail.com

1 Ulsan Ship and Ocean College,

Ludong University, Yantai, China

\section{Springer}

\begin{abstract}
In this article we study applications of the Schrödinger-type identity for obtaining transmutations via the fixed point index for nonlinear integral equations. It is possible to derive a wide range of transmutation operators by this method. Classical Riesz transforms are involved in the Schrödinger-type identity method as basic blocks, among them are Fourier, sine and cosine-Fourier, Hankel, Mellin, Laplace, and some generalized transforms. In this paper, we present a modified Schrödinger-type identity for solutions of a class of linear Schrödinger equations with mixed boundary conditions. The techniques used in our proofs are quite different, and most remarkably some of the proofs become simpler and more straightforward. As an application, we obtain the existence and uniqueness of a solution to the stationary Schrödinger equation in the sense of the Weyl law, which advances the recent results obtained in several articles even in a more general setting.
\end{abstract}

Keywords: Schrödinger-type identity; Neumann boundary condition; Stationary Schrödinger equation

\section{Introduction}

The stationary Schrödinger equations are often used to express real-life problems. Naturally, we focus on finding solutions of linear Schrödinger equations. However, analytical solutions are frequently not possible to find and numerical solutions can be both theoretically and computationally complicated due to the complexity of a modified Schrödinger operator. Frequently, researchers investigate inequalities in weighted Banach spaces; see, for example, [1-6] and the references therein for details. Hence, it is appropriate to derive a generalized stationary Schrödinger inequality equation from modified Schrödinger differential equations (see $[4,5,7]$ ).

In recent years, under the frame of the auxiliary principle, some references, such as Ding [8], Noor [7], Chen and Zhang [9], Huang [10], and so on, introduced the related properties of weak solutions for generalized stationary Schrödinger equations of fluid dynamics and built the corresponding convergence theorems.

Due to the rapid advancement of computing resource, rigorous derivation of generalized stationary Schrödinger equations was done also for Schrödinger fluids. An asymptotic analysis of the generalized Schrödinger differential equation based on the asymptotic

(c) The Author(s) 2019. This article is distributed under the terms of the Creative Commons Attribution 4.0 International License (http://creativecommons.org/licenses/by/4.0/), which permits unrestricted use, distribution, and reproduction in any medium, provided you give appropriate credit to the original author(s) and the source, provide a link to the Creative Commons license, and indicate if changes were made. 
expansion was presented in $[9,10]$. In this paper we investigate a new equation of generalized stationary Schrödinger inequalities and prove an existence and uniqueness theorem of solutions for this kind of equation.

The rest of the paper is organized as follows. In Sect. 2, we present some basic definitions, concepts, and some results that will be used later for our model problem. In Sect. 3, we prove the existence and uniqueness of weak solutions for generalized stationary Schrödinger equations. Finally, the paper is concluded in Sect. 4.

\section{Preliminaries}

In 2013, Ren [11] proposed the following linear Schrödinger model:

$$
\begin{aligned}
& \frac{d S}{d t}=r(S+I)\left(1-\frac{S+I}{K}\right)-\beta S I-\eta \gamma_{1}(S) Y, \\
& \frac{d I}{d t}=\beta S I-\gamma(I) Y-C I, \\
& \frac{d Y}{d t}=\left(\varepsilon \gamma(I)+\eta \varepsilon \gamma_{1}(S)-d\right) Y,
\end{aligned}
$$

where $S$ is the number of sound prey, $I$ is the number of infected prey population, $Y$ is the number of predator population, $\gamma(I)$ and $\eta \gamma_{1}(S)$ are predator functional response functions. Ren analyzed model (1) in terms of positivity, uniqueness, boundedness and studied the existence of the Hopf bifurcation.

Model (1) may be re-written by Wang, Mai, and Wang (see [12]) in a simplified form as follows:

$$
\begin{aligned}
& \frac{d S}{d t}=r S\left(1-\frac{S+I}{K}\right)-\beta S I, \\
& \frac{d I}{d t}=-c I+\beta S I-p I Y, \\
& \frac{d Y}{d t}=-d Y+p q I Y .
\end{aligned}
$$

Let $I=\{1,2\}$ be an index set, $H_{i}$ be a real Hilbert space with inner product $\langle\cdot, \cdot\rangle_{i}$ and norm $\|\cdot\|_{i}$, respectively, where $i=1$, 2. Let $A: H_{1} \rightarrow H_{1}, B: H_{2} \rightarrow H_{2}, F_{1}: H_{1} \times H_{2} \rightarrow H_{1}$, and $\eta_{1}: H_{1} \times H_{1} \rightarrow H_{1}$ be mappings. Let $a_{i}: H_{i} \times H_{i} \rightarrow \mathbb{R}$ be a coercive continuous map such that

(C1) $a_{i}\left(\sigma_{i}, \sigma_{i}\right) \geq c_{i}\left\|\sigma_{i}\right\|_{i}^{2}$;

(C2) $\left|a_{i}\left(\varrho_{i}, \sigma_{i}\right)\right| \leq d_{i}\left\|\varrho_{i}\right\|_{i} \cdot\left\|\sigma_{i}\right\|_{i}$ for any $\varrho_{i}, \sigma_{i} \in H_{i}$.

Let $b_{i}: H_{i} \times H_{i} \rightarrow \mathbb{R}$ be a map with nondifferentiable terms such that

(C3) $b_{i}$ is a linear function for the first variable;

(C4) $b_{i}$ is a convex function;

(C5) There exists a positive constant $\gamma_{i}$ satisfying

$$
\gamma_{i}\left\|\varrho_{i}\right\|_{i} \cdot\left\|\sigma_{i}\right\|_{i} \geq a_{i}\left(\varrho_{i}, \sigma_{i}\right)
$$

for any $\varrho_{i}, \sigma_{i} \in H_{i}$. 


$$
b_{i}\left(\varrho_{i}, \sigma_{i}-w_{i}\right) \geq b_{i}\left(\varrho_{i}, \sigma_{i}\right)-b_{i}\left(\varrho_{i}, w_{i}\right)
$$

for any $\varrho_{i}, \sigma_{i}, w_{i} \in H_{i}$.

Based on the above notations, we then define the proposed equation of generalized nonlinear stationary Schrödinger inequality problems as follows (see [13, 14]):

$$
\begin{aligned}
\frac{\partial F_{1}(x, y)}{\partial x}= & F_{1}(x, y)\left[b_{1}\left(x, \sigma_{1}\right)-\eta_{1}(x, y)\left(F_{1}(x, y)+F_{2}(x, y)\right)-a_{1}(x, y) f_{1}\left(x, \sigma_{1}-x\right)\right] \\
\frac{\partial F_{2}(x, y)}{\partial x}= & F_{2}(x, y)\left[b_{1}\left(x, \sigma_{1}\right)-\eta_{2}(x, y)\left(F_{1}(x, y)+F_{2}(x, y)\right)-a_{2}(x, y) f_{2}\left(x, \sigma_{2}-x\right)\right] \\
\frac{\partial f_{i}(x, y)}{\partial x}= & -d(x, y) x_{3}(x, y)-b(x, y) x_{3}^{2}(x, y)+c_{1}(x, y) x_{3}(t-\tau) F_{1}(t-\tau) \\
& +c_{2}(x, y) x_{3}(t-\tau) F_{2}(t-\tau) \quad(i=1,2)
\end{aligned}
$$

where $F_{1}, F_{2}$, and $f_{i}$ are susceptible, infected, and predator population respectively, and the corresponding parameters have the meaning as defined in [15]. Time delay is considered as a gestation period, and a disease can be transmitted by contact and spreads among prey species only.

Remark 1 There are some special cases for the model problem (3):

(1) If $A=B=I, f_{i}=0$, and $a_{i}\left(\varrho_{i}, \sigma_{i}\right)=0$, then (3) is equivalent to

$$
\left\langle F_{1}(x, y), \eta_{1}\left(\sigma_{1}, x\right)\right\rangle_{1}+b_{1}\left(x, \sigma_{1}\right)-b_{1}(x, x) \geq 0
$$

for any $\sigma_{1} \in H_{1}$ and

$$
\left\langle F_{2}(x, y), \eta_{2}\left(\sigma_{2}, y\right)\right\rangle_{2}+b_{2}\left(y, \sigma_{2}\right)-b_{2}(y, y) \geq 0
$$

for any $\sigma_{2} \in H_{2}$.

(2) If $H_{1}=H_{2}=H, f_{i}=f_{2}=f, \eta_{1}=\eta_{2}=\eta, a_{1}=a_{2}=a, b_{1}=b_{2}=b$, then (3) is reduced to

$$
\langle F(A x, B x)-f, \eta(w, y)\rangle+a(y, w-y)+b(y, w)-b(y, y) \geq 0
$$

for any $v \in H$.

We then introduce the following definition (see $[16,17])$, which will be useful for the proposed method.

Definition 1 Let $A: H_{1} \rightarrow H_{1}, B: H_{2} \rightarrow H_{2}, F_{1}: H_{1} \times H_{2} \rightarrow H_{1}$, and $\eta_{1}: H_{1} \times H_{1} \rightarrow H_{1}$ be mappings. Then

(1) $F_{1}$ is $\alpha_{1}$-strongly monotone if the following inequality holds:

$$
\left.\left\langle F_{1}\left(A \varrho_{1}, \varrho_{2}\right)-F_{1}\left(A \sigma_{1}, \varrho_{2}\right), \varrho_{1}-\sigma_{1}\right)\right\rangle_{1} \geq \alpha_{1}\left\|\varrho_{1}-\sigma_{1}\right\|_{1}^{2}
$$

where $\varrho_{1}, \sigma_{1} \in H_{1}, \varrho_{2} \in H_{2}$ and $\alpha_{1}$ is a positive constant; 
(2) If there exist two positive constants $\beta_{1}$ and $\xi_{1}$ such that

$$
\left\|F_{1}\left(A \varrho_{1}, B \varrho_{2}\right)-F_{1}\left(A \sigma_{1}, B \sigma_{2}\right)\right\|_{1} \leq \beta_{1}\left\|\varrho_{1}-\sigma_{1}\right\|_{1}+\xi_{1}\left\|\varrho_{2}-\sigma_{2}\right\|_{2}
$$

for $\varrho_{1}, \sigma_{1} \in H_{1}$ and $\varrho_{2}, \sigma_{2} \in H_{2}$, then $F_{1}$ is $\left(\beta_{1}, \xi_{1}\right)$-Lipschitz continuous;

(3) If

$$
\left\|\eta_{1}\left(\varrho_{1}, \sigma_{1}\right)\right\|_{1} \leq \delta_{1}\left\|\varrho_{1}-\sigma_{1}\right\|_{1}
$$

for $\varrho_{1}, \sigma_{1} \in H_{1}$, then $\eta_{1}$ is $\delta_{1}$-Lipschitz continuous, where $\delta_{1}$ is a positive constant.

(4) If

$$
\left\langle\varrho_{1}-\sigma_{1}, \eta_{1}\left(\varrho_{1}, \sigma_{1}\right)\right\rangle_{1} \geq \sigma_{1}\left\|\varrho_{1}-\sigma_{1}\right\|_{1}^{2}
$$

for $\varrho_{1}, \sigma_{1} \in H_{1}$, then $\eta_{1}$ is $\sigma_{1}$-strongly monotone, where $\sigma_{1}$ is a positive constant.

\section{Existence and uniqueness}

In this section, we give an existence and uniqueness theorem of the solution of the auxiliary problem for (3). Under the frame of this theorem, we develop a new linearization iterative algorithm for the proposed model. First of all, let us present the following auxiliary problem for (3).

It follows that there exist $z_{1} \in H_{1}$ and $z_{2} \in H_{2}$ such that (see [18])

$$
\begin{aligned}
& \left\langle z_{1}-\eta_{1}, \eta_{1}\left(\sigma_{1}, z_{1}\right)\right\rangle_{1}+\rho_{1}\left\langle F_{1}\left(A \eta_{1}, B x_{2}\right)-f_{1}, \eta_{1}\left(\sigma_{1}, z_{1}\right)\right\rangle_{1}+\rho_{1}\left[a_{1}\left(z_{1}, \sigma_{1}-z_{1}\right)\right] \\
& \quad+\rho_{1}\left[b_{1}\left(\eta_{1}, \sigma_{1}\right)-b_{1}\left(\eta_{1}, z_{1}\right)\right] \geq 0
\end{aligned}
$$

for any $\sigma_{1} \in H_{1}$.

$$
\begin{aligned}
& \left\langle z_{2}-x_{2}, \eta_{2}\left(\sigma_{2}, z_{2}\right)\right\rangle_{2}+\rho_{2}\left\langle F_{2}\left(A \eta_{1}, B x_{2}\right)-f_{2}, \eta_{2}\left(\sigma_{2}, z_{2}\right)\right\rangle_{2}+\rho_{2}\left[a_{2}\left(z_{2}, \sigma_{2}-z_{2}\right)\right] \\
& \quad+\rho_{2}\left[b_{2}\left(x_{2}, \sigma_{2}\right)-b_{1}\left(x_{2}, z_{2}\right)\right] \geq 0
\end{aligned}
$$

for any $\sigma_{2} \in H_{2}$ and any given $\left(\eta_{1}, x_{2}\right) \in H_{1} \times H_{2}$.

By applying a fixed point theorem, which is due to Yao et al. (see [19]), we have the following.

Theorem 1 Let $\phi_{i}$ be a continuous function defined in $H_{1} \times H_{2}$ and $S$ be multifunctions defined in $\mathbb{R}_{+}$. If there exists a function $m \in \mathcal{L}_{\text {loc }}^{1}\left(\mathbb{R}_{+}\right)$satisfying

$$
m(s) \geq H(F(s, x, y), 0)
$$

for any $t \in \mathbb{R}_{+}$, then there exists a solution $x$ of problem (3) satisfying

$$
\left|\eta_{1}(s)-\rho_{2}(s)\right| \leq\left(\left|\eta_{1}(0)-x_{0}\right|+\int_{0}^{L} \rho_{1}(s) d s\right) \exp \left(2 \lambda^{+} s\right)
$$

for $s \in[0, L]$, where $\lambda^{+}=\max \{\lambda, 0\}$. 


\section{Proof Define}

$$
\psi_{i}(s, \varsigma, \beta)=\left\{x \in F(s, \varsigma, \beta):\left\langle\dot{x}_{1}(s)-x, \eta_{1}(s)-\varsigma\right\rangle \leq \lambda\left|\eta_{1}(s)-\varsigma\right|^{2}\right\} .
$$

It is easy to see that it is nonempty for any $s \in[0, L]$. So

$$
\left|\dot{\eta}_{1}(s)-w\right|=d\left(\dot{x}_{1}(s), \phi_{i}\left(s, \eta_{1}(s), \max _{s \in S(s)} \eta_{1}(s)\right)\right) \leq \rho_{1}(s),
$$

which together with (C3) gives that

$$
\left\langle w-x, \eta_{1}(s)-\varsigma\right\rangle \leq \lambda\left|\eta_{1}(s)-\varsigma\right|^{2}+\left|\eta_{1}(s)-\varsigma\right| .
$$

So

$$
\begin{aligned}
& \left\langle\dot{x}_{1}(s)-x, \eta_{1}(s)-\varsigma\right\rangle \\
& \quad \leq\left\langle w-x, \eta_{1}(s)-\varsigma\right\rangle+\left|\dot{x}_{1}(s)-w\right|\left|\eta_{1}(s)-\varsigma\right| \\
& \quad \leq \lambda\left|\eta_{1}(s)-\varsigma\right|^{2}+\left|\eta_{1}(s)-\varsigma\right|\left(\lambda\left|\max _{s \in S(s)} \eta_{1}(s)-\beta\right|+\rho_{1}(s)\right),
\end{aligned}
$$

which yields that there exists a solution $x$ of problem

$$
\begin{aligned}
& \dot{x}(s) \in G\left(s, \rho_{2}(s), \max _{s \in S(s)} x(s)\right), \\
& x(0)=x_{0}
\end{aligned}
$$

satisfying

$$
\begin{aligned}
& \left\langle\dot{x}_{1}(s)-\dot{x}(s), \eta_{1}(s)-\rho_{2}(s)\right\rangle \\
& \quad \leq \lambda\left|\eta_{1}(s)-\rho_{2}(s)\right|^{2}+\left|\eta_{1}(s)-\rho_{2}(s)\right|\left(\lambda\left|\max _{s \in S(s)} \eta_{1}(s)-\max _{s \in S(s)} x(s)\right|+\rho_{1}(s)\right) \\
& \quad \leq \lambda\left|\eta_{1}(s)-\rho_{2}(s)\right|^{2}+\left|\eta_{1}(s)-\rho_{2}(s)\right|\left(\lambda \max _{s \in S(s)}\left|\eta_{1}(s)-x(s)\right|+\rho_{1}(s)\right)
\end{aligned}
$$

for any $s \in[0, L]$.

Define

$$
r(s)=\max _{s \in S(s)}\left|\eta_{1}(s)-\rho_{2}(s)\right|,
$$

which is absolutely continuous and differentiable. It follows that

$$
r(s) \dot{r}(s)=\frac{d}{d t} r^{2}(s) \leq \lambda r(s)\left(r(s)+\max _{s \in S(s)} r(s)\right)
$$

from (8).

And define

$$
T=\{s \in[0, L]: r(s)=0\} .
$$


It follows that

$$
\dot{r}(s) \leq \lambda^{+}\left(r(s)+\max _{s \in S(s)} r(s)\right)+\rho_{1}(s)
$$

for any $t \notin T$ from (9), which yields that $\bar{r}$ is the solution of

$$
\begin{aligned}
& \dot{\bar{r}}(s)=\lambda^{+}\left(\bar{r}(s)+\max _{s \in S(s)} \bar{r}(s)\right)+\rho_{1}(s), \quad s \in[0, L] \\
& \bar{r}(0)=r(0) .
\end{aligned}
$$

So

$$
\bar{r}(s) \leq r(0)+\int_{0}^{s}\left(2 \lambda^{+} \bar{r}(\tau)+\rho_{1}(\tau)\right) d \tau,
$$

which implies the required conclusion.

\section{Theorem 2 Let the following conditions hold:}

(1) $a_{i}: H_{i} \times H_{i} \rightarrow \mathbb{R}$ satisfy (C1) and (C2), $b_{i}: H_{i} \times H_{i} \rightarrow \mathbb{R}$ with (C3)-(C6);

(2) $\eta_{i}$ is $\sigma_{i}$-strongly monotone;

(3) $\left\langle F_{i}(A x, B y), \eta_{i}\left(\sigma_{i}, \cdot\right)\right\rangle$ is concave and upper semicontinuous.

Then the auxiliary problems (4) and (5) are solvable.

Proof Let mappings $\phi_{i}, \psi_{i}: H_{i} \times H_{i} \rightarrow \mathbb{R}$ be defined as follows:

$$
\phi_{i}\left(\sigma_{i}, z_{i}\right)=\left\langle\sigma_{i}-x_{i}, \eta_{i}\left(\sigma_{i}, z_{i}\right)\right\rangle_{i}+\rho_{i}\left\langle F_{i}\left(A \eta_{1}, B x_{2}\right)-f_{i}, \eta_{i}\left(\sigma_{i}, z_{i}\right)\right\rangle_{i}
$$

and

$$
\psi_{i}\left(\sigma_{i}, z_{i}\right)=\left\langle z_{i}-x_{i}, \eta_{i}\left(\sigma_{i}, z_{i}\right)\right\rangle_{i}+\rho_{i}\left\langle F_{i}\left(A \eta_{1}, B x_{2}\right)-f_{i}, \eta_{i}\left(\sigma_{i}, z_{i}\right)\right\rangle_{i},
$$

respectively.

So

$$
\begin{aligned}
\phi_{i}\left(\sigma_{i}, z_{i}\right)-\psi_{i}\left(\sigma_{i}, z_{i}\right) & =\left\langle\sigma_{i}-z_{i}, \eta_{i}\left(\sigma_{i}, z_{i}\right)\right\rangle_{i}+\rho_{i}\left[a_{i}\left(\sigma_{i}-z_{i}, \sigma_{i}-z_{i}\right)\right] \\
& \geq\left(\sigma_{i}+\rho_{i} c_{i}\right)\left\|\sigma_{i}-z_{i}\right\|_{i}^{2} \geq 0
\end{aligned}
$$

for any $\sigma_{i}, z_{i} \in H_{i}$, which yields that 1 (1) holds. And $a_{i}$ is coercive continuous, then $a_{i}\left(\sigma_{i}, \sigma_{i}-z_{i}\right)$ is weakly upper semicontinuous.

It follows that $b_{i}$ is convex and lower semicontinuous, which means that $\left\langle F_{i}(A x, B y)\right.$, $\left.\eta_{i}\left(\sigma_{i}, \cdot\right)\right\rangle$ is concave and upper semicontinuous. Hence, $\phi_{i}\left(\sigma_{i}, \cdot\right)$ and $\left\{\sigma_{i} \in H_{i}: \psi_{i}\left(\sigma_{i}, z_{i}\right)\right\}$ are convex. That is to say, conditions (2) and (3) hold.

Furthermore,

$$
w_{i}=\left[\sigma_{i}+\rho_{i} c_{i}\right]^{-1}\left\{\rho_{i}\left\|F_{i}\left(A \eta_{1}, B x_{2}\right)-f_{i}\right\|_{i}+\left(\rho_{1 i}+\rho_{i} \gamma_{i}\right)\left\|x_{i}\right\|_{i}\right\}
$$


and

$$
K_{i}=\left\{z_{i} \in H_{i}:\left\|z_{i}\right\|_{i} \leq w_{i}\right\}
$$

for each $i \in I$.

So

$$
\begin{aligned}
\psi_{i}\left(\sigma_{0_{i}}, z_{i}\right)= & \psi_{i}\left(0, z_{i}\right) \\
= & \left\langle z_{i}-x_{i}, \eta_{i}\left(0, z_{i}\right)\right\rangle_{i}+\rho_{i}\left\langle F_{i}\left(A \eta_{1}, B x_{2}\right)-f_{i}, \eta_{i}\left(0, z_{i}\right)\right\rangle_{i} \\
& +\rho_{i}\left[a_{i}\left(z_{i}, 0-z_{i}\right)\right] \\
= & -\left\langle 0-z_{i}, \eta_{i}\left(0, z_{i}\right)\right\rangle_{i}+\left\langle x_{i}, \eta_{i}\left(z_{i}, 0\right)\right\rangle_{i}+\rho_{i}\left\langle F_{i}\left(A \eta_{1}, B x_{2}\right)-f_{i}, \eta_{i}\left(0, z_{i}\right)\right\rangle_{i} \\
& -\rho_{i}\left[a_{i}\left(z_{i}, z_{i}\right)\right] \\
\leq & -\sigma_{i}\left\|z_{i}\right\|_{i}^{2}+\rho_{1 i}\left\|x_{i}\right\|_{i}\left\|z_{i}\right\|_{i}+\rho_{i}\left\|F_{i}\left(A \eta_{1}, B x_{2}\right)-f_{i}\right\|_{i}\left\|z_{i}\right\|_{i} \\
& -\rho_{i} c_{i}\left\|z_{i}\right\|_{i}^{2}+\rho_{i} \gamma_{i}\left\|x_{i}\right\|_{i}\left\|z_{i}\right\|_{i} \\
= & -\left\|z_{i}\right\|_{i}\left\{\left(\sigma_{i}+\rho_{i} c_{i}\right)\left\|z_{i}\right\|_{i}-\rho_{i}\left\|F_{i}\left(A \eta_{1}, B x_{2}\right)-f_{i}\right\|_{i}-\left(\rho_{1 i}+\rho_{i} \gamma_{i}\right)\left\|x_{i}\right\|_{i}\right\} \\
\leq & 0 .
\end{aligned}
$$

There exists $z_{i}^{*} \in H_{i}$ such that

$$
\left\langle\sigma_{i}-x_{i}, \eta_{i}\left(\sigma_{i}, z_{i}^{*}\right)\right\rangle_{i}+\rho_{i}\left\langle F_{i}\left(A \eta_{1}, B x_{2}\right)-f_{i}, \eta_{i}\left(\sigma_{i}, z_{i}^{*}\right)\right\rangle_{i}+\rho_{i}\left[a_{i}\left(\sigma_{i}, \sigma_{i}-z_{i}^{*}\right)\right] \geq 0
$$

for any $s \in(0,1]$ and $\sigma_{i} \in H_{i}$ from Theorem 1 .

If $\sigma_{i}$ is replaced by $y_{i, t}$ in (11), then we obtain that

$$
\begin{aligned}
0 \leq & \left\langle y_{i, t}-x_{i}, \eta_{i}\left(y_{i, t}, z_{i}^{*}\right)\right\rangle_{i}+\rho_{i}\left\langle F_{i}\left(A \eta_{1}, B x_{2}\right)-f_{i}, \eta_{i}\left(y_{i, t}, z_{i}^{*}\right)\right\rangle_{i} \\
& +\rho_{i}\left[a_{i}\left(y_{i, t}, y_{i, t}-z_{i}^{*}\right)\right]+\rho_{i}\left[b_{i}\left(x_{i}, y_{i, t}\right)\right] \\
= & -\left\langle y_{i, t}-x_{i}, \eta_{i}\left(z_{i}^{*}, y_{i, t}\right)\right\rangle_{i}-\rho_{i}\left\langle F_{i}\left(A \eta_{1}, B x_{2}\right)-f_{i}, \eta_{i}\left(z_{i}^{*}, y_{i, t}\right)\right\rangle_{i} \\
& +\rho_{i}\left[a_{i}\left(y_{i, t}, y_{i, t}-z_{i}^{*}\right)\right]+\rho_{i}\left[b_{i}\left(x_{i}, y_{i, t}\right)\right] \\
\leq & -t\left\langle y_{i, t}-x_{i}, \eta_{i}\left(z_{i}^{*}, \sigma_{i}\right)\right\rangle_{i}-\rho_{i} t\left\langle F_{i}\left(A \eta_{1}, B x_{2}\right)-f_{i}, \eta_{i}\left(z_{i}^{*}, \sigma_{i}\right)\right\rangle_{i},
\end{aligned}
$$

where

$$
y_{i, t}:=t \sigma_{i}+(1-t) z_{i}^{*}
$$

In fact, in the above formulation, the second inequality comes from that $\eta_{i}$ is affine in the second variable, $\eta_{i}\left(z_{i}^{*}, z_{i}^{*}\right)=0$ and $b_{i}$ satisfies $(\mathrm{C} 3)-(\mathrm{C} 6)$.

Hence, we derive

$$
\left\langle y_{i, t}-x_{i}, \eta_{i}\left(\sigma_{i}, z_{i}^{*}\right)\right\rangle_{i}+\rho_{i}\left\langle F_{i}\left(A \eta_{1}, B x_{2}\right)-f_{i}, \eta_{i}\left(\sigma_{i}, z_{i}^{*}\right)\right\rangle_{i}+\rho_{i}\left[a_{i}\left(y_{i, t}, \sigma_{i}-z_{i}^{*}\right)\right] \geq 0,
$$


which yields that

$$
\begin{aligned}
& \left\langle z_{i}^{*}-x_{i}, \eta_{i}\left(\sigma_{i}, z_{i}^{*}\right)\right\rangle_{i}+\rho_{i}\left(F_{i}\left(A \eta_{1}, B x_{2}\right)-f_{i}, \eta_{i}\left(\sigma_{i}, z_{i}^{*}\right)\right\rangle_{i}+\rho_{i}\left[a_{i}\left(z_{i}^{*}, \sigma_{i}-z_{i}^{*}\right)\right] \\
& \quad+\rho_{i}\left[b_{i}\left(x_{i}, \sigma_{i}\right)-b_{i}\left(x_{i}, z_{i}^{*}\right)\right] \geq 0
\end{aligned}
$$

in the case $t \rightarrow 0^{+}$.

Therefore, $z_{1}^{*} \in H_{1}$ and $z_{2}^{*} \in H_{2}$ are the solutions of auxiliary problems (4) and (5), respectively.

In the following, we prove the uniqueness of problems (4) and (5). For any two solutions $z_{1}, z_{1}^{\prime} \in H_{1}$ of (4), we have

$$
\begin{aligned}
& \left\langle z_{1}-\eta_{1}, \eta_{1}\left(\sigma_{1}, z_{1}\right)\right\rangle_{1}+\rho_{1}\left\langle F_{1}\left(A \eta_{1}, B x_{2}\right)-f_{1}, \eta_{1}\left(\sigma_{1}, z_{1}\right)\right\rangle_{1}+\rho_{1}\left[a_{1}\left(z_{1}, \sigma_{1}-z_{1}\right)\right] \\
& \quad+\rho_{1}\left[b_{1}\left(\eta_{1}, \sigma_{1}\right)-b_{1}\left(\eta_{1}, z_{1}\right)\right] \geq 0 \quad \forall \sigma_{1} \in H_{1}, \\
& \left\langle z_{1}^{\prime}-\eta_{1}, \eta_{1}\left(\sigma_{1}, z_{1}^{\prime}\right)\right\rangle_{1}+\rho_{1}\left\langle F_{1}\left(A \eta_{1}, B x_{2}\right)-f_{1}, \eta_{1}\left(\sigma_{1}, z_{1}^{\prime}\right)\right\rangle_{1}+\rho_{1}\left[a_{1}\left(z_{1}^{\prime}, \sigma_{1}-z_{1}^{\prime}\right)\right] \\
& \quad+\rho_{1}\left[b_{1}\left(\eta_{1}, \sigma_{1}\right)-b_{1}\left(\eta_{1}, z_{1}^{\prime}\right)\right] \geq 0 \quad \forall \sigma_{1} \in H_{1} .
\end{aligned}
$$

Taking $\sigma_{1}=z_{1}^{\prime}$ in (12) and $\sigma_{1}=z_{1}$ in (13), we obtain that

$$
\begin{aligned}
& \left\langle z_{1}-\eta_{1}, \eta_{1}\left(z_{1}^{\prime}, z_{1}\right)\right\rangle_{1}+\rho_{1}\left\langle F_{1}\left(A \eta_{1}, B x_{2}\right)-f_{1}, \eta_{1}\left(z_{1}^{\prime}, z_{1}\right)\right\rangle_{1}+\rho_{1}\left[a_{1}\left(z_{1}, z_{1}^{\prime}-z_{1}\right)\right] \\
& \quad+\rho_{1}\left[b_{1}\left(\eta_{1}, z_{1}^{\prime}\right)-b_{1}\left(\eta_{1}, z_{1}\right)\right] \geq 0, \\
& \left\langle z_{1}^{\prime}-\eta_{1}, \eta_{1}\left(z_{1}, z_{1}^{\prime}\right)\right\rangle_{1}+\rho_{1}\left\langle F_{1}\left(A \eta_{1}, B x_{2}\right)-f_{1}, \eta_{1}\left(z_{1}, z_{1}^{\prime}\right)\right\rangle_{1}+\rho_{1}\left[a_{1}\left(z_{1}^{\prime}, z_{1}-z_{1}^{\prime}\right)\right] \\
& \quad+\rho_{1}\left[b_{1}\left(\eta_{1}, z_{1}\right)-b_{1}\left(\eta_{1}, z_{1}^{\prime}\right)\right] \geq 0 .
\end{aligned}
$$

Adding (14) and (15), we deduce that

$$
\begin{aligned}
\sigma_{1}\left\|z_{1}-z_{1}^{\prime}\right\|_{1}^{2} & \leq\left\langle z_{1}-z_{1}^{\prime}, \eta_{1}\left(z_{1}, z_{1}^{\prime}\right)\right\rangle_{1} \\
& \left.\leq-\rho_{1}\left[a\left(z_{1}-z_{1}^{\prime}\right), z_{1}-z_{1}^{\prime}\right)\right] \\
& \leq-\rho_{1} c_{1}\left\|z_{1}-z_{1}^{\prime}\right\|_{1}^{2},
\end{aligned}
$$

which yields $z_{1}=z_{1}^{\prime}$. That is to say, $z_{1}^{*} \in H_{1}$ is the unique solution of (4). In a similar fashion, $z_{2}^{*} \in \mathrm{H}_{2}$ is the unique solution of (5).

\section{Conclusions}

In this work, we have studied applications of the Schrödinger-type identity for obtaining transmutations via the fixed point index for nonlinear integral equations. It was possible to derive a wide range of transmutation operators by this method. Classical Riesz transforms were involved in the Schrödinger-type identity method as basic blocks, among them are Fourier, sine and cosine-Fourier, Hankel, Mellin, Laplace, and some generalized transforms. In this paper, we presented a modified Schrödinger-type identity for solutions of a class of linear Schrödinger equations with mixed boundary conditions. The techniques used in our proofs were quite different, and most remarkably some of the proofs became simpler and more straightforward. As an application, we obtained the existence 
and uniqueness of a solution to the stationary Schrödinger equation in the sense of the Weyl law, which advanced the recent results obtained in several articles even in a more general setting.

\section{Acknowledgements}

The author is very grateful to the editor and referees for their useful suggestions, which have improved the paper.

\section{Funding}

Not applicable.

\section{Abbreviations}

Not applicable

Availability of data and materials

Not applicable.

\section{Competing interests}

The author declares that he has no competing interests.

\section{Authors' contributions}

The author read and approved the final manuscript.

\section{Publisher's Note}

Springer Nature remains neutral with regard to jurisdictional claims in published maps and institutional affiliations.

Received: 25 December 2018 Accepted: 13 March 2019 Published online: 21 March 2019

\section{References}

1. Cottle, R.: Nonlinear programs with positively bounded Jacobians. Ph.D. Dissertation, Department of Mathematics, University of California, Berkeley (1964)

2. Glowinski, R., Lions, J., Trémolières, R.: Numerical Analysis of Variational Inequalities. North-Holland, Amsterdam (1981)

3. Hartman, P., Stampacchia, G.: On some nonlinear elliptic differential functional equations. Acta Math. 115, 153-188 (1966)

4. Lions, J., Stampaccia, G.: Variational inequalities. Commun. Pure Appl. Math. 20, 493-512 (1967)

5. Panagiotopoulos, P.: Inequality Problems in Mechanics and Applications. Birkhäuser, Boston (1985)

6. Parida, J., Sen, A.: A variational-like inequality problems for multi-functions with applications. J. Math. Anal. Appl. 124, 73-81 (1987)

7. Noor, M.: Mixed variational-like inequalities. Commun. Appl. Nonlinear Anal. 1, 63-75 (1994)

8. Ding, X.: Algorithm of solutions for mixed-nonlinear variational-like inequalities in reflexive Banach space. Appl. Math. Mech. 19, 521-529 (1998)

9. Zhang, X., Liu, D., Yan, Z., Zhao, G., Yuan, Y.: Schrödinger-type identity for Schrödinger free boundary problems. Bound. Value Probl. 2018, 135 (2018)

10. Shi, D.: Upper bounds on the number of eigenvalues of stationary Schrödinger operators. J. Math. Phys. 51(8), Article ID $083523(2010)$

11. Ren, Y.: Solving integral representations problems for the stationary Schrödinger equation. Abstr. Appl. Anal. 2013, Article ID 715252 (2013)

12. Wang, J., Mai, A., Wang, H.: Existence and uniqueness of solutions for the Schrödinger integrable boundary value problem. Bound. Value Probl. 2018, 74 (2018)

13. Lions, P.L.: Mathematical Topics in Fluid Mechanics. Volume 2: Compressible Models. Oxford Lecture Series in Mathematics and Its Applications, vol. 10. Clarendon, Oxford (1998)

14. Krasnosel'skii, M.A., Rutickii, J.B.: Convex Functions and Orlicz Spaces. Noordhoff, Groningen (1961)

15. Qiao, Y., Guo, H.: Green matrix of eigenvalue problem and completeness of eigenfunction systems for a Hamiltonian operator derived from the wave equations. J. Inn. Mong. Univ. Nat. Sci. 49, 113-119 (2018)

16. Kufner, A., John, O., Fučík, S.: Function Spaces. Monographs and Textbooks on Mechanics of Solids and Fluids. Mechanics: Analysis. Noordhoff, Leyden (1977)

17. Nazarov, S.A.: Asymptotic solution of the Navier-Stokes problem on the flow of a thin layer of fluid. Sib. Math. J. 31(2) 296-307 (1990)

18. Chang, S., Xiang, S.: On the existence of solutions for a class of quasi-bilinear variational inequality. J. Syst. Sci. Math. Sci. 16, 136-140 (1996)

19. Yao, Y., Zhang, W., Wen, B., Kang, J., Ahmed, A.: Stationary solutions of a class of nonlinear Schrödinger equations. Acta Anal. Funct. Appl. 19(2), 119-124 (2017) 